\title{
Clinical macrovascular disease in Caucasoid diabetic subjects: logistic regression analysis of risk variables
}

\author{
T.A. Welborn, M. Knuiman, V. McCann, K. Stanton and I.J.Constable \\ Departments of Medicine, Ophthalmology and Mathematics, University of Western Australia, Nedlands, Western Australia
}

Summary. A cross-sectional study of 1084 Caucasoid diabetic subjects in rural Western Australia revealed a high rate of clinical macrovascular disease $(46 \%)$, including coronary heart disease $(13 \%)$, stroke $(8 \%)$, and peripheral vascular disease $(38 \%)$. Age was the major time-related variable for total macrovascular disease and for peripheral vascular disease, with identical prevalence rates in Type 1 (insulin-dependent) and Type 2 (non-insulin-dependent) diabetes when age was taken into account. In 179 Type 1 diabetic subjects, logistic regression analysis showed no associated risk factors other than age. In 905 Type 2 diabetic subjects the independent risk factors for total macrovascular disease, identified by a forward stepwise selection procedure, were age as the major contributor, with plasma creatinine levels and plasma glucose levels (all $p<0.001$ ), high-density lipoprotein cholesterol levels, serum total cholesterol levels, and the (supine-erect) systolic blood pressure difference $($ all $p<0.05)$. There were no direct associations with percentage desirable weight, cigarette smoking or male sex. Type 2 diabetic subjects demonstrated a very strong negative association between high-density lipoprotein cholesterol levels and coronary heart disease, and significant associations were found also between plasma glucose levels and coronary heart disease $(p<0.01)$, and glycosylated haemoglobin levels and peripheral vascular disease $(p<0.001)$.

Key words: Diabetes, macrovascular disease, risk factors.
Occlusive atherosclerotic vascular disease is recognised as being the most common complication of diabetes today [1, 2]. The International Atherosclerosis Project [3] showed that the prevalence of the large vessel lesions in diabetic patients parallels the prevalence in the natural population, but that the former have more extensive raised lesions and more complicated lesions than nondiabetic subjects. Studies in the United States and England [4-6] have confirmed the substantial increase in mortality from coronary heart disease in subjects with diabetes and with impaired glucose tolerance, and that the habitual protection of females from atherosclerosis is abolished. Multivariate analyses demonstrate that the conventional risk factors in the population operate also for diabetic patients [4-6]. The independent relationships of blood glucose levels and of diabetic control to macrovascular disease have not been clearly established.

We report here the frequency of clinically-defined macrovascular disease in cross-sectional surveys of Caucasoid diabetic subjects in rural Western Australia. Plasma glucose levels and glycosylated haemoglobin levels were measured as an indication of diabetic control in additon to conventional cardiovascular risk fac- tors, and the relevance of these variables has been assessed by the statistical technique of logistic regression analysis.

\section{Subjects and methods}

\section{Subjects}

The diabetic subjects were voluntary respondents to surveys held in major country centres $50-800 \mathrm{~km}$ from Perth, Western Australia, in 1978-1982 [7], for the purpose of screening for the ophthalmic and vascular complications of diabetes. The centres were Kalgoorlie, Busselton, Geraldton, Albany, Bunbury, Northam, Esperance, Mandurah, Narrogin, Port Hedland, Merredin and Moora. From demographic data provided by the Australian Bureau of Census and Statistics (total population 103,000) and with knowledge of the prevalence of known diabetes in Australia [8], it is estimated that approximately $70 \%$ of the diabetic subjects available in the area attended. A total of 1218 persons were screened, of whom 1084 were Caucasoid. The data from 134 subjects of Aboriginal or part-Aboriginal race were analysed separately.

Macrovascular disease was ascertained as follows: coronary heart disease: symptoms of definite angina pectoris and of definite past myocardial infarction were identified using the London School of Hygiene questionnaire [9]. Definite angina pectoris or myocardial infarction implies "doctor-confirmed" in that when positive responses were given to the questionnaire, a history also had to be obtained that 
Table 1. The number of diabetic subjects studied, by type and treatment, and the rates of clinical macrovascular disease and its sub-categories

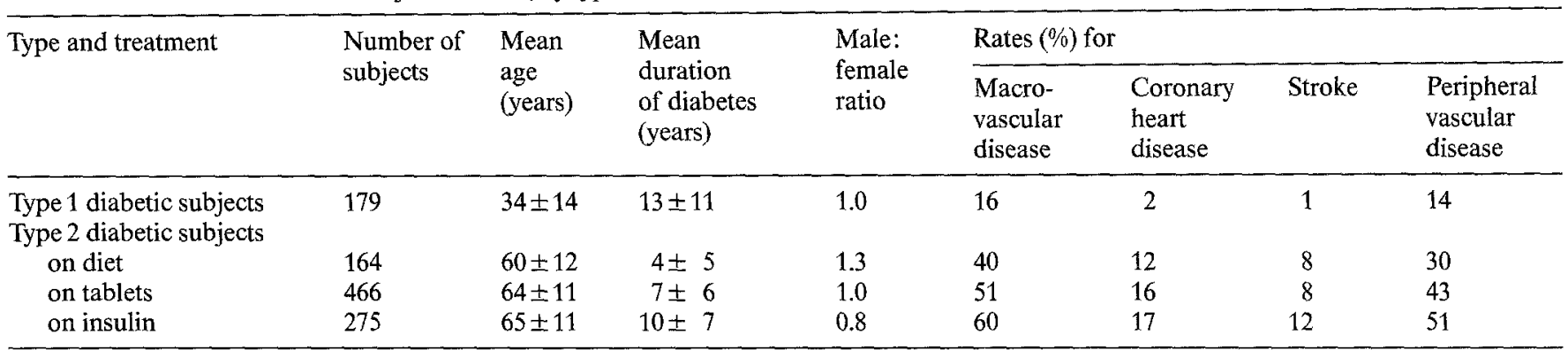

Time-related variables expressed as mean \pm SD

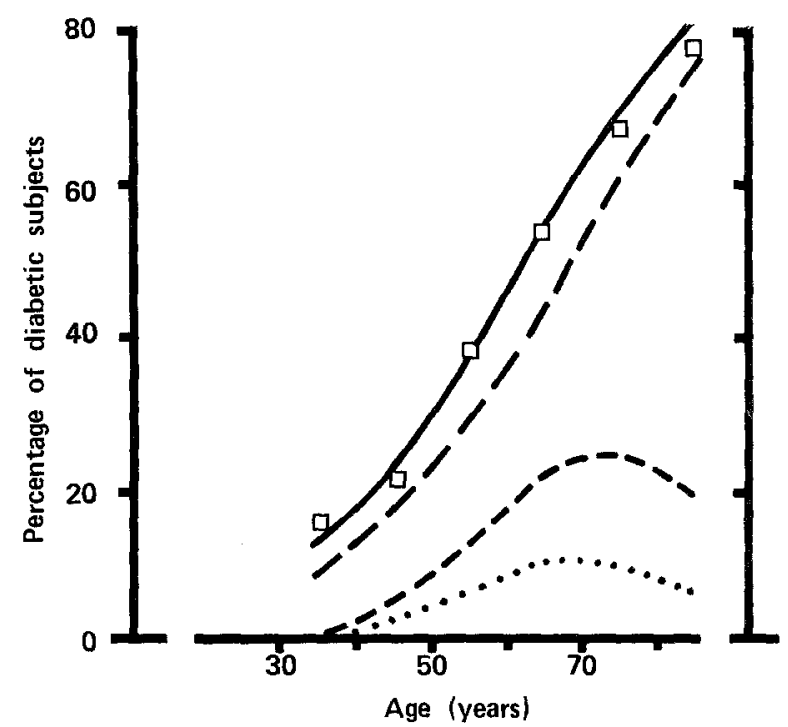

Type 2 diabetes

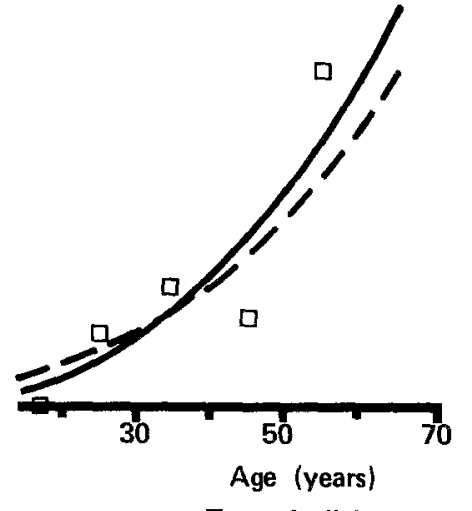

Type 1 diabetes
Fig. 1. The estimated prevalence rates by logistic modelling for total macrovascular disease (-), peripheral vascular disease $(----)$, coronary heart disease $(-\ldots-\ldots)$, and stroke $(. . .$.$) by age in$ Type 2 and Type 1 diabetic subjects. The open squares indicate the observed prevalence rates for total macrovascular disease the patient had seen his doctor about the chest pain, and the doctor had given a cardiac diagnosis [10]. In rural Western Australia this technique improves the specificity of the questionnaire, $71 \%$ having ECG changes ( $65 \%$ frank, $6 \%$ borderline) compatible with coronary heart disease [10]. The chest pain questionnaire is a valid technique for identifying a disease-rich group within one population and for demonstrating the association of coronary heart disease with major risk factors [11]. Cerebrovascular disease was identified by a positive answer to the enquiry "have you ever had a stroke?" and required confirmation by the interviewing physician. Peripheral vascular disease was identified by a standard questionnaire for lower limb claudication administered by a physician, or was ascertained if two or more foot pulses were judged absent by the physician.

The subjects attended between 0900 and $1700 \mathrm{~h}$ without reference to mealtimes or medications. Type 1 diabetic subjects were defined as those with age at onset of diabetes $<40$ years, requiring permanent insulin therapy within 2 years of diagnosis. All remaining subjects were classified as Type 2 diabetes. This method of classification segregates the two types of diabetes into low- and high-serum-C-peptide classes with $93 \%$ accuracy overall [12]. The examination included measurement of blood pressure after $10 \mathrm{~min}$ rest, supine and erect, using mercury sphygmomanometers. Height and weight were recorded and the body mass index (weight $(\mathrm{kg}) \div$ height $^{2}(\mathrm{~m})$ ) was calculated. Current smoking habits and current medications were recorded.

Non-fasting blood samples were obtained and were processed immediately for transport to the city-based teaching-hospital laboratories for analyses. These were: glycosylated haemoglobin levels by the centrifuge-accelerated method of Davis and Nicol [13]; total plasma cholesterol by cholesterol oxidase $[14,15]$; serum high-density lipoprotein-cholesterol (HDL-cholesterol) by the method of Burstein and Samaille [16]. Plasma glucose was measured by hexokinase using glucose test reagents (SVR, Calbiochem-Behring, Cat no 870104) and plasma creatinine by autoanalyser (Technicon RA 1000 system, Technicon Instruments, Tarrytown, New York, USA).

A logistic regression method [17] was used to determine the strength of association of possible risk factors with macrovascular disease. The importance of each variable under consideration was assessed by the significance of the contribution that it made to the deviance of the logistic regression. The deviance is a measure of the discrepancy between the fitted values derived from the logistic regression equation and the observed values. With all diabetic complications, a time-related variable is usually of importance, such as age of onset, or duration, or actual age. Accordingly, the logistic regression is performed for each variable when considered alone and also after allowing for the contribution of the major time-related variable. Finally, a forward stepwise procedure is used to select the subset of risk variables which best explains the presence of the complication by independent association.

The data used for analysis by multiple regression were complete in most respects, except that the HDL-cholesterol values were performed in 672 of the 1084 subjects. The missing values were randomly distributed throughout the diabetic population in that the corresponding samples were not obtained from certain towns (Busselton, Bunbury, Northam). As the forward stepwise procedure used to select the best subset of independent risk variables requires the data to be complete, estimates of the missing HDL-cholesterol values were used for this part of the analysis. These estimates were obtained from a multiple linear regression of HDL-cholesterol on related measurements derived from the available data. Careful checks for possible bias were performed, and in addition the final logistic equation obtained by the forward stepwise procedure were shown to have very similar coefficients when fitted to the actual data of 672 subjects. For simplicity of presentation, the results of all forward stepwise procedures quoted relate to analyses that include the estimated HDL-cholesterol values. 
Table 2. Multiple logistic regression analysis of variables associated with diabetic macrovascular disease in Type 1 and Type 2 diabetic subjects. The deviance contributions for each variable are considered alone and after allowing for the effect of age. The forward stepwise selection procedure indicates the independent relationship of risk variables to macrovascular disease prevalance

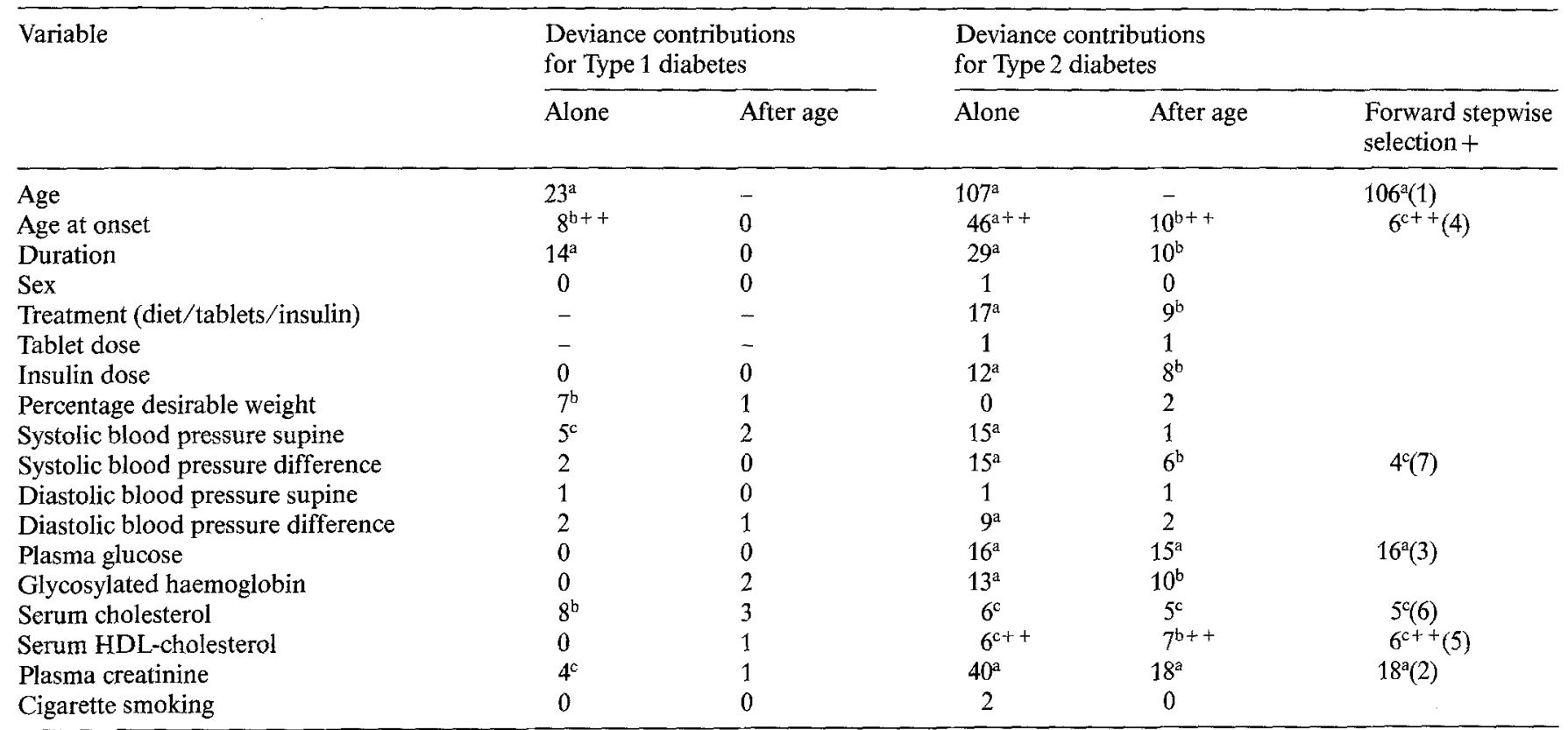

There were no interactions between test variables. + Order of selection in parentheses; ++ indicates a negative association. ${ }^{a}$ Denotes signifi-

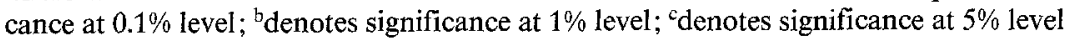

Table 3. Prevalence $(P)$ of macrovascular disease in Type 2 diabetic subjects. Coefficients for the fitted logistic equation for prevalence of macrovascular disease in Type 2 diabetic subjects (after forward stepwise selection procedure, Table 2) and magnitude of influence of each risk variable over the range mean $\pm \mathrm{SD}$

\begin{tabular}{|c|c|c|}
\hline $\begin{array}{l}\text { Fitted logistic equation for } \\
\operatorname{logit}(P)=\log \frac{(P)}{}=\end{array}$ & $\begin{array}{l}\text { Range of mean } \pm S D \\
\text { for risk variables }\end{array}$ & $\begin{array}{l}\text { Influence on } \\
\text { prevalence }(\%)\end{array}$ \\
\hline-6.302 (constant) & & $\ldots$ \\
\hline$+0.093 \times$ age (years) & $\ddot{53}-75$ & +47 \\
\hline $\begin{array}{l}-0.000312 \times \text { onset age } \\
\left(\text { years }^{2}\right)\end{array}$ & $44-68$ & -20 \\
\hline $\begin{array}{l}+0.011 \times \text { plasma creatinine } \\
\quad(\mu \mathrm{mol} / \mathrm{l})\end{array}$ & $60-120$ & +16 \\
\hline $\begin{array}{l}-0.628 \times \text { plasma } \\
\text { HDL-cholesterol } \\
(\mathrm{mmol} / \mathrm{l})\end{array}$ & $0.9-1.6$ & -12 \\
\hline $\begin{array}{l}+0.046 \times \text { plasma glucose } \\
(\mathrm{mmol} / \mathrm{l})\end{array}$ & $5.4-14.6$ & +10 \\
\hline $\begin{array}{l}+0.125 \times \text { plasma cholesterol } \\
(\mathrm{mmol} / \mathrm{l})\end{array}$ & $4.9-7.6$ & +8 \\
\hline $\begin{array}{l}+0.0101 \times \text { systolic blood } \\
\text { pressure difference } \\
(\mathrm{mm} . \mathrm{Hg})\end{array}$ & $-8.0-+22$ & +8 \\
\hline
\end{tabular}

\section{Results}

The prevalence of clinical macrovascular disease by type of diabetes and treatment class is shown in Table 1. There is no marked sex difference. The discrepancy in prevalence between Type 1 and Type 2 diabetes may clearly be related to age. Within the therapeutic classes of Type 2 diabetes there appear to be differences in prevalence, possibly age-related. Overall the prevalence of macrovascular disease was $46 \%$, including $13 \%$ with coronary heart disease, $8 \%$ with stroke and $38 \%$ with peripheral vascular disease.

When examined by logistic modelling, age rather than duration or age at onset was the major time-related variable associated with macrovascular disease. The fitted prevalence rates for peripheral vascular disease and total macrovascular disease increase progressively with age (Fig. 1), and at any given age the rates for Type 1 and Type 2 diabetic subjects are almost identical, and can be superimposed. In Type 2 diabetic subjects the prevalence rates for coronary heart disease and stroke plateau after age 60 then fall, probably an indication of selective mortality. In Type 1 diabetic subjects no significant age relationship was found with coronary heart disease and stroke, these complications showing very low prevalence rates $(1-2 \%)$.

The risk factors for all macrovascular disease were analysed by multiple logistic regression for Type 1 and Type 2 diabetic subjects separately, and are shown in Table 2. In Type 1 diabetic subjects the marked effect of age is seen, and significant associations occur with other variables when considered alone, including serum cholesterol and percentage desirable weight. After age was accounted for, none of the remaining variables showed any significant independent association with macrovascular disease.

In Type 2 diabetic subjects the profound relationship of macrovascular disease with age is demonstrated again (Table 2). Many of the remaining variables retain significant associations with macrovascular disease 
Table 4. Type 2 diabetic subjects: risk variables for categories of clinical macrovascular disease by forward stepwise logistic regression analysis, in order of selection. The levels of significance are obtained from $t$-values for the coefficient for each variable in the final logistic equation

\begin{tabular}{|c|c|c|}
\hline Coronary heart disease & Stroke & $\begin{array}{l}\text { Peripheral vascular } \\
\text { disease }\end{array}$ \\
\hline Plasma HDL-cholesterol ${ }^{a \dagger}$ & $\mathrm{Age}^{b}$ & $\operatorname{Age}^{\mathrm{a}}$ \\
\hline $\operatorname{Age}^{a}$ & Age $\times \operatorname{age}^{b f}$ & Plasma creatinine $\mathrm{a}^{\mathrm{a}}$ \\
\hline Age $\times$ age $^{a \dagger}$ & & $\begin{array}{l}\text { Glycosylated } \\
\text { haemoglobin }\end{array}$ \\
\hline Plasma glucose ${ }^{b}$ & & $\begin{array}{l}\text { Diastolic blood } \\
\text { pressure difference } \\
\text { Age at onset }\end{array}$ \\
\hline
\end{tabular}

${ }^{a}$ Denotes significance at $0.1 \%$ level; ${ }^{b}$ denotes significance at $1 \%$ level; ${ }^{c}$ denotes significance at $5 \%$ level. $\nmid$ Indicates a negative association

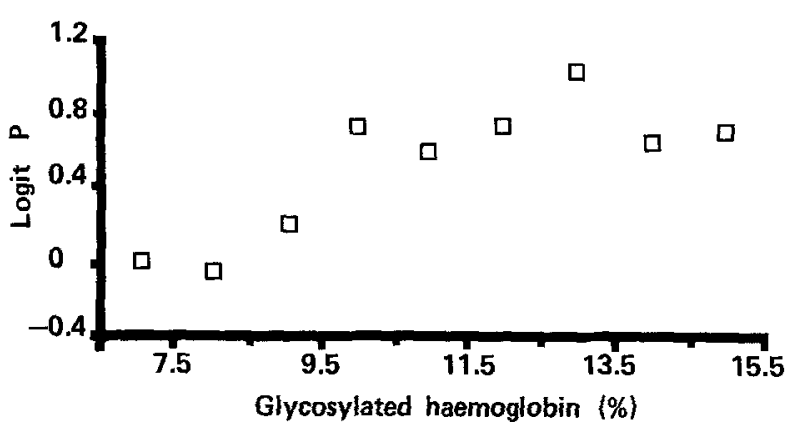

Fig. 2. The relationship of logit $P$ (where $P$ is prevalence of macrovascular disease in Type 2 diabetic subjects) to glycosylated haemoglobin levels in the logistic regression equation after age has been accounted for. No statistical evidence could be found for a non-linear (threshold) phenomenon

prevalence after age is taken into account. Thus there appears to be a significant relationship with treatment category (diet, oral hypoglycaemic agents, or insulin therapy) and with insulin dosage (in Type 2 diabetic subjects) even after the age effect is removed. However, the forward stepwise selection procedure reveals the subset of variables that best 'explain' the presence of macrovascular disease by their independent contributions to the regression equation. Thus, in order of selection, age, plasma creatinine and plasma glucose levels have highly significant deviance contributions $(p<$ 0.001 ), followed by age at onset (negative association), serum HDL-cholesterol (negative association), serum cholesterol, and the supine-erect systolic blood pressure difference (all $p<0.05$ ).

The actual coefficients for the fitted logistic equation for prevalence of macrovascular disease in Type 2 diabetic subjects are listed in Table 3, to show the magnitude of 'influence' of each risk variable over the range mean $\pm S D$. Even after the substantial effects of age, all of the remaining factors contribute materially to the prevalence figure. Their association with macrovascular disease appears to be biologically as well as statistically relevant.
The risk factors for the individual categories of macrovascular disease were analysed by logistic regression. and showed some striking differences from the preceding results in Type 2 diabetes. (In Type 1 diabetic subjects, after age, not one of the remaining variables showed any significant association with any category of macrovascular disease). The analyses for Type 2 diabetic subjects are summarised (Table 4) by listing the results of the forward stepwise selection procedures in order. Age continues to be a major determinant in all cases, but the relationship with age is quadratic for coronary heart disease and stroke (as may be inferred from Fig.1). For coronary heart disease, HDL-cholesterol levels supercede age in order of selection. For stroke, age and age $x$ age are the only significant related variables.

An index of diabetic control, either plasma glucose or glycosylated haemoglobin levels, is a significant associated factor in coronary heart disease and peripheral vascular disease, as well as total macrovascular disease. These two measures of control are closely inter-related. The relationship of glycosylated haemoglobin to categories of macrovascular disease was carefully examined to determine whether prevalence was related in a linear manner or showed a 'threshold' phenomenon, and an example is shown in Figure 2. In this example the statistical model embodying separate effects for each glycosylated haemoglobin class provided no better fit than the linear model, and no convincing evidence for a 'threshold' phenomenon was found despite extended analysis.

'Systolic blood pressure difference' (supine-erect) and 'diastolic blood pressure difference' (supine-erect) were independently associated with all macrovascular disease and peripheral vascular disease respectively in Type 2 diabetic subjects, rather than the actual blood pressure levels. In this group the mean blood pressure levels were $161 / 87 \mathrm{mmHg}$ supine, $149 / 87 \mathrm{mmHg}$ erect. The blood pressure difference (supine-erect) has been found to be the independently-related variable for retinopathy and vision-threatening retinopathy [7] as well as neuropathy and nephropathy (M. Knuiman, unpublished data). This phenomenon is unexplained. Systolic and diastolic blood pressure levels, although closely correlated with 'blood pressure difference' could not be 'forced' into the regression equation by omission of the latter. Those on anti-hypertensive therapy comprised $49 \%$ of Type 2 diabetic subjects. They showed no significant differences in the extent or distribution of postural hypotension as compared with those on no therapy.

Twenty-three percent of the study group were current cigarette smokers; one half exceeded 130\% desirable weight; and one half were male sex. These factors as candidate variables were consistently rejected by the forward stepwise selection procedure as having an independent association with macrovascular disease prevalence in either Type 1 or Type 2 diabetes. 


\section{Discussion}

The very high prevalence of symptomatic macrovascular disease described, and the close relationship with age, reinforce the view that in any particular population diabetes has an additive effect on the pathogenesis of macrovascular disease [19]. In this cross-sectional study of a diabetic population we hoped to discern possible aetiological relationships between risk factors and the clinical manifestations of atherosclerotic disease. For Type 1 diabetic subjects, total macrovascular disease and peripheral vascular disease showed age-related prevalence rates strikingly similar to those in Type 2 diabetic subjects, but no variables other than age were related independently to these complications, and none was related to coronary heart disease or stroke. Further analysis of larger samples is necessary to determine macrovascular disease risk factors in Type 1 diabetes.

In contrast, an array of significant, independentlyassociated variables was found in Type 2 diabetic subjects, and interesting differences in the risk factor profiles emerged with the analysis of separate categories of macrovascular disease. The study demonstrates an independent relationship between level of diabetic control in Type 2 diabetic subjects and the prevalence of macrovascular disease. Much evidence suggests that diabetes and impaired glucose tolerance enhance the expression of atherosclerosis, especially coronary heart disease [1-6]. In addition prospective studies of (nondiabetic) populations have related subsequent coronary heart disease mortality to a threshold value of the $2-\mathrm{h}$ plasma glucose during baseline glucose tolerance; the plasma glucose values following $50-\mathrm{g}$ and $75-\mathrm{g}$ glucose loads being $5.3 \mathrm{mmol} / 1$ in the Whitehall study [5] and $7.8 \mathrm{mmol} / 1$ in the Paris study [18], respectively. In our prevalence study, no threshold phenomenon could be determined statistically, but it is clear (Fig. 2) that an increase in the risk of macrovascular disease is apparent above a glycosylated haemoglobin value of $9.5 \%$, which corresponds to mean plasma glucose levels of 7-8 mmol/1 [20].

The role of lipid metabolism in diabetic atherosclerosis has been inferred from the observed tendency for diabetic subjects to have elevated cholesterol and triglyceride levels [21] and to show low HDL-cholesterol values in some but not all studies [22]. In Framingham [4] the high incidence of coronary heart disease in diabetic women was attributed in part to their very low HDLcholesterol levels. An age-matched comparison of diabetic subjects from the present study with subjects from a normal rural population in Western Australia has shown significantly lower HDL-cholesterol values in the diabetic group [15] and proportionately lower HDLcholesterol values in diabetic females. We can extend these findings therefore to link high cholesterol levels and low HDL-cholesterol levels to macrovascular disease in an exclusively diabetic population, but especial- ly to link low HDL-cholesterol levels to coronary heart disease in Type 2 diabetic subjects.

Elevated blood pressure levels in diabetic subjects have been shown by multivariate analysis to determine subsequent coronary heart disease [4-6]. The lack of any such association in the present study may relate to the high proportion of subjects on anti-hypertensive therapy. However, the emergence of postural hypotension ('systolic-diastolic blood pressure difference') as an independently related risk factor in Type 2 diabetes, for peripheral vascular disease especially, does not appear to relate to drug therapy. It may indicate vascular sclerosis or less likely the occurrence of autonomic neuropathy.

Cigarette smoking also is a recognized risk factor for subsequent cardiovascular events in diabetic subjects [5, 6]. Our failure to demonstrate any correlation between current smoking and vascular disease may well reflect the crudity of a simple smoking questionnaire in indicating actual exposure to the risk. The lack of association of smoking and cardiovascular disease is a wellknown artefact of cross-sectional studies.

Elevated plasma creatinine levels ranked highly as an associated independent factor for peripheral vascular disease and total macrovascular disease in Type 2 diabetic subjects. Possibly this reflects diabetic and/or hypertensive nephropathy and the tendency for macroand microvascular disease to go hand in hand.

At this stage it is reasonable to interpret our and others' data as indicating that all conventional risk factors for vascular disease require scrupulous attention in diabetic subjects, including blood lipids, blood pressure and cigarette smoking. In the prevention of atherosclerotic complications, effective intervention with the nondiabetic cardiovascular risk factors may prove to be more feasible than the control of hyperglycaemia. Although diabetes and impaired glucose tolerance are substantial predictive risk factors [1-6] and our data support the possibility of an independent relationship of 'diabetic control' to macrovascular disease, there is no good evidence yet to confirm that improvement in diabetic control diminishes the risk of macrovascular disease. If such an experiment is conducted, the available data $[5,18]$ suggest that 'near normal' glucose tolerance will be necessary to reverse such risk.

Acknowledgement. This study was supported by the Diabetes Research Foundation of Western Australia, the Lions Save Sight Foundation, and the TVW Telethon Foundation. We are grateful for assistance and advice from Drs. G. Stein, R. Yuen, C. Glatthaar, D. Greer and R. Cooper. Dr. R. E. Davis performed the glycosylated haemoglobin measurements and Dr. J. R. Maserei the lipoprotein assays.

\section{References}

1. Jarrett RJ, Keen H, Chakrabarti R (1982) Diabetes hyperglycaemia and arterial disease. In: Keen $H$, Jarrett $R$ (eds) Complications of diabetes, 2nd ed. Arnold, London, pp. 179-184

2. West KM (1978) Epidemiology of diabetes and its vascular lesions. Elsevier, New York, pp.353-369 
3. Robertson WB, Strong JP (1968) Atherosclerosis in persons with hypertension and diabetes mellitus. Lab Invest 18: 538-551

4. Kannel WB, McGee DL (1979) Diabetes and glucose tolerance as risk factors for cardiovascular disease: the Framingham study. Diabetes Care 2: 120-126

5. Fuller JH, Shipley MJ, Rose G, Jarrett RJ, Keen H (1980) Coronary heart disease risk and impaired glucose tolerance. Lancet 1 : 1373-1376

6. Jarrett RJ, McCartney P, Keen H (1982) The Bedford survey: ten year mortality rates in newly diagnosed diabetics, borderline diabetics and normoglycaemic controls and risk indices for coronary heart disease in borderline diabetics. Diabetologia 22: 79-84

7. Constable IJ, Knuiman MW, Welborn TA, Cooper RL, Stanton KM, McCann VJ, Gross JC (1984) Assessing the risk of diabetic retinopathy. Am J Ophthalmol 97: 53-61

8. National Heart Foundation of Australia (1980) Risk factor prevalence study report No 1. National Heart Foundation of Australia, Canberra, pp. 54-55

9. Rose G, Blackburn H (1968) Cardiovascular survey methods. WHO Monograph Series No 56, Geneva

10. Welborn TA, Cumpston GN, Cullen KJ, Curnow DH, McCall MG, Stenhouse NS (1969) The prevalence of coronary heart disease and associated factors in an Australian rural community. Am J Epidemiol 59:521-536

11. Rose G, Keen H, Jarrett RJ (1979) Epidemiologic methods in diabetic macrovascular disease. Diabetes Care 2:91-97

12. Welborn TA, Garcia-Webb P, Bonser A, McCann V, Constable IJ (1983) Clinical criteria that reflect C-peptide status in idiopathic diabetes. Diabetes Care 6:315-316

13. Davis RE, Nicol DJ (1978) A rapid simplified method for the routine measurement of glycosylated haemoglobin. Lancet 2: 350-351

14. Allain CC, Poon LS, Chan CSG, Richmond W, Fu PC (1974) Enzymatic determination of total serum cholesterol. Clin Chem 20: $470-475$
15. Maserei JRL, Constable IJ, Stanton K, Davis RE (1982) Cholesterol and lipoprotein-cholesterol levels in Western Australian rural diabetics. Aust NZ J Med 12: 241-247

16. Burstein M, Samaille J (1960) Rapid estimation of lipoprotein cholesterol in serum. Clin Chim Acta 5: 609

17. Alvey NG, Banfield CF, Baxter RI, Gower JC, Krzanowski WJ, Lane PW, Leech PK, Nelber JA, Payne RW, Thelps KM, Rodger CW, Ross GJS, Simpson HR, Todd AD, Wedderburn RWM, Wilkinson GN (1977) GENSTAT: A general statistical programme. Rothamstead Experimental Station, Rothamstead, UK, pp. 7p1-7p6

18. Eschwege E, Ducimetiere P, Rapaz L, Claude JR, Richard JL (1980) Blood glucose and coronary heart disease. Lancet 2: $472-473$

19. Keen H, Jarrett RJ (1979) The WHO multinational study of vascular disease in diabetics: macrovascular disease prevalence. Diabetes Care 2: 187-195

20. Welborn TA, Knuiman M, Davis RE, Stanton K, McCann V, Constable I (1983) Applying the correlation between glycosylated haemoglobin and plasma glucose values. Diabetologia 24: 461-462 (Letter)

21. Lewis B (1976) The hyperlipidaemias. Blackwell, Oxford, London, pp. 294-303

22. Nikkila EA (1981) High density lipoproteins in diabetes. Diabetes 30 (Suppl 2): 82-87

Received: 4 November 1983

and in revised form: 26 September 1984

Dr. T. A. Welborn

Department of Endocrinology and Diabetes

Queen Elizabeth II Medical Centre

Perth 6009

Western Australia 